Citation: Demir, H., Sari, R., "Riemannian Submersions With Quarter- Symmetric Non-metric Connection". Journal of Engineering Technology and Applied Sciences 6 (1) 2021 : 1-8.

\title{
RIEMANNIAN SUBMERSIONS WITH QUARTER- SYMMETRIC NON-METRIC CONNECTION
}

\author{
Hakan Demir ${ }^{a}$ (D), Ramazan Sari ${ }^{\mathbf{b} *}$ (iD) \\ ${ }^{a}$ Alaca Avni Çelik Vocational Schools, Hitit University \\ hakandemir@hitit.edu.tr \\ ${ }^{b}$ Gümüşhacıköy Hasan Duman Vocational Schools, Amasya University \\ ramazan.sari@amasya.edu.tr (*corresponding author)
}

\begin{abstract}
In this paper, we study Riemannian submersions from a Riemannian manifold endowed with a quartersymmetric non-metric connection onto a Riemannian manifold. We investigate O'Neill's tensor fields for quarter-symmetric non-metric connection and derive the covariant derivative of O'Neill's tensor fields. We obtain derivatives of those tensor fields and compare curvatures of the total manifold, the base manifold, and the fibers by computing curvatures.
\end{abstract}

Keywords: Riemannian submersions, quarter symmetric connection, O’Neill’s tensors

\section{Introduction}

Friedmann et all defined the concept of the semi-symmetric non-metric connection in a differential manifold [1]. Later, Hayden studied metric connection with torsion a Riemannian manifold [2]. Afterward, Yano investigated a Riemannian manifold with a new connection called a semi-symmetric metric connection [3]. Many authors have studied semi-symmetric connections [4-6].

Let $M$ be differentiable manifold with lineer connection $\nabla$. Therefore for all $X, Y \in \Gamma(T M)$

$T(X, Y)=\nabla_{X} Y-\nabla_{Y} X-[X, Y]$

where $T$ is torsion tensor of $\nabla$. If $T=0$, therefore the connection $\nabla$ is said to be symmetric, otherwise it is called non-symmetric. 
In 1975, Golab introduced quarter symmetric connection in a diferentiable manifold [7]. A linear connection $\nabla$ is said to be quarter symmetric connection if its torsion tensor $T$ is given by

$T(X, Y)=\eta(Y) \varphi(X)-\eta(X) \varphi(Y)$

where $\varphi$ is a tensor field of type $(1,1)$ and $\eta$ is a 1 -form. Moreover, the quarter symmetric connection reduces to a semi-symmetric connection. Because quarter symmetric connection generalizes the semi symmetric connection. Many authors have studied the quarter symmetric connection [8-11].

One way to compare two manifolds is to define smooth maps from one manifold to another. One of these maps is submersion, the rank of the map is equal to the dimension of the target manifold. Riemannian submersion between Riemannian submanifolds was first introduced by O’ Neill and Gray $[12,13]$.

The idea of semi-symmetric non-metric connections in a Riemannain submersion was introduced by Akyol and Beyendi [14]. They investigated O’Neill's tensor fields, obtain derivatives of those tensor fields and compare curvatures of the total manifold, the base manifold and the fibres by computing curvatures.

In this paper, we investigate Riemannian submersion with quarter symmetric non-metric connection. We investigate basic properties of Riemannian submersion with quarter symmetric non-metric connection.

\section{Preliminaries}

Let $\pi:\left(M^{m}, g_{M}\right) \rightarrow\left(B^{b}, g_{B}\right)$ be a submersion between two Riemannian manifolds. Then $\pi$ said to be Riemannian submersion if

i) $\pi$ has maximal rank

ii) The diferantial $\pi_{*}$ preserves the lengths of horizontal vector.

On the other hand, $\pi^{-1}(k)$ is an $(m-b)$ dimensional submanifold of $M$, For each $k \in M$ The submanifolds $\pi^{-1}(k)$ are called fibers. Moreover, vector fields tangent to fibers are called vertical and vector fields orthogonal to fibers are horizantal. A vector field $X$ on $M$ is called basic if $X$ is horizontal and $\pi_{*} X_{p}=X_{\pi_{*(q)}}^{\prime}$ for all $q \in M$. We determine that $V$ and $H$ define projections $k e r \pi_{*}$ and $\left(k e r \pi_{*}\right)^{\perp}$, respectively.

On the other hand, a Riemannian submersion $\pi: M \rightarrow B$ determines tensor fields $T$ and $A$ on $M$ such that,

$$
\begin{aligned}
& T(E, F)=T_{E} F=h \nabla_{v E} v F+v \nabla_{v E} h F \\
& A(E, F)=A_{E} F=v \nabla_{h E} h F+h \nabla_{h E} v F
\end{aligned}
$$

for any $E, F \in \Gamma(T M)$. By virtue of (1) and (2), we have

$\nabla_{V} W=T_{V} W+\widehat{\nabla}_{V} W$,

$\nabla_{V} X=T_{V} X+h\left(\nabla_{V} X\right)$, 
$\nabla_{X} V=v\left(\nabla_{X} V\right)+A_{X} V$

$\nabla_{X} V=A_{X} Y+h\left(\nabla_{X} Y\right)$

for any $X, Y \in \Gamma\left(k e r \pi_{*}\right)^{\perp}$ and $V, W \epsilon \Gamma\left(k e r \pi_{*}\right)$. Moreover, if $X$ is basic therefore

$h\left(\nabla_{V} X\right)=A_{X} V$.

$A_{X} Y=\frac{1}{2} v[X, Y]$ refecting the complete integrability of the horizontal distribution $H$. It is known that $A$ is alternating on the horizontal distribution, such that $A_{X} Y=-A_{Y} X$ for $X, Y \in \Gamma\left(k e r \pi_{*}\right)^{\perp}$ and $T$ is symetric on the vertical distribution.

Now, we define a linear connection on a Riemannian manifold $M$ by

$\widetilde{\nabla}_{X} Y=\nabla_{X} Y+\eta(X) \varphi Y$

where $X, Y \in \Gamma(T M), \nabla$ is Levi-Civita connection on $M, \eta$ is a 1 -form associated with the vector field $P$ on $M$ by $\eta(Y)=g_{M}(Y, P)$ and $\varphi$ is $(1,1)$ - type tensor field.

On the other hand, the torsion tensor $\widetilde{T}$ with respect to the connection $\widetilde{\nabla}$ is

$\widetilde{\mathrm{T}}_{X} Y=\widetilde{\nabla}_{X} Y-\widetilde{\nabla}_{Y} X-[X, Y]$

for all $X, Y \in \Gamma(T M)$.

Then, using (3) and $\nabla$ is Levi-Civita connection, we have

$\widetilde{\mathrm{T}}_{X} Y=\eta(Y) \varphi X-\eta(X) \varphi Y$

Moreover, for Riemannian metric $g$, from (3) we get,

$\left(\widetilde{\nabla}_{X} g\right)(Y, Z)=\eta(Y) g(\varphi X, Z)+\eta(Z) g(Y, \varphi X)$

for all $X, Y \in \Gamma(T M)$. A linear connection $\widetilde{\nabla}$ defined by (3) satisfies (4) and (5) and hence we call $\widetilde{\nabla}$ is quarter-symmetric non-metric connection.

On the other hand, let $\pi: M \rightarrow B$ be a Riemannian submersion betwen two Riemannian manifolds and $\widetilde{\nabla}$ is a quarter symmetric non-metric connection. Therefore, tensors $\widetilde{T}$ and $\widetilde{A}$ with type (1.2) on $M$ with respect to $\widetilde{\nabla}$ isgiven by

$\widetilde{\mathrm{T}}_{E} F=h \widetilde{\nabla}_{v E} v F+v \widetilde{\nabla}_{v E} h F$

and

$\widetilde{\mathrm{A}}_{E} F=v \widetilde{\nabla}_{h E} h F+h \widetilde{\nabla}_{h E} v F$. 


\section{Riemannian submersions with a quarter-symmetric non-metric connection}

We note that, for brevity we use a abbreviation " $\pi$ is a Riemannian submersion with QSNMC" for $\pi: M \rightarrow B$ is a Riemannian submersion between two Riemannian manifolds with quartersymmetric non-metric connection $\widetilde{\nabla}$ on $M$.

Theorem 3.1. Let $\pi$ is a Riemannian submersion with QSNMC. Then the tensor $T$ and $A$ with type (1.2) on $M$ with respect to $\widetilde{\nabla}$ is given by

$\widetilde{\mathrm{T}}_{E} F=T_{E} F+\eta(v F) h \varphi(v E)+\eta(h F) v \varphi(v E)$

and

$\widetilde{\mathrm{A}}_{E} F=A_{E} F+\eta(h F) v \varphi(h E)+\eta(v F) h \varphi(h E)$

for all $E, F \in \Gamma(T M)$.

Proof. For all $E, F \in \Gamma(T M)$ using (6) and (3) we get,

$$
\widetilde{\mathrm{T}}_{E} F=h \nabla_{v E} v F+\eta(v F) h \varphi(v E)+v \nabla_{v E} h F+h(h F) v \varphi(v E) .
$$

Then we using (1), which gives (8).

On the other hand for all $E, F \in \Gamma(T M)$ using (2) and (3)

$$
\widetilde{\mathrm{A}}_{E} F=v \nabla_{h E} h F+(h F) v \varphi(h E)+h \nabla_{h E} v F+h(v F) h \varphi(h E) .
$$

After some calculate we have proof the theorem.

Theorem 3.2 Let $\pi$ is a Riemannian submersion with QSNMC. Then we have

$$
\begin{aligned}
g\left(\widetilde{\mathrm{T}}_{E} F, G\right)= & g\left(\widetilde{\mathrm{T}}_{E} G, F\right)+2 g\left(T_{E} F, G\right)+\eta(v F) g(h \varphi(v E), G) \\
& +\eta(h F) g(v \varphi(v E), G)-\eta(v G) g(h \varphi(v E), F)-\eta(h G) g(V \varphi(v E), v F)
\end{aligned}
$$

and

$$
\begin{aligned}
g\left(\widetilde{\mathrm{A}}_{E} F, G\right)= & g\left(A_{E} G, F\right)+2 g\left(A_{E} F, G\right)+\eta(h F) g(v \varphi(h E), v G) \\
& -\eta(h G) g(v \varphi(h E), v F)+\eta(v F) g(h \varphi(h E), h G)-\eta(v G) g(h \varphi(h E), h F)
\end{aligned}
$$

for all $E, F, G \in \Gamma(T M)$.

Proof. For all $E, F, G \in \Gamma(T M)$, by using (8) we get,

$$
g\left(\widetilde{\mathrm{T}}_{E} F, G\right)=g\left(T_{E} G, F\right)+\eta(v F) g(h \varphi(v E), G)+(h F) g(v \varphi(v E), G) .
$$

We know that,

$$
G=v G+h G .
$$


Then, we have

$g\left(\widetilde{\mathrm{T}}_{E} F, G\right)=g\left(T_{E} F, G\right)+\eta(v F) g(h \varphi(v E), G)+\eta(h F) g(v \varphi(v E), G)$

In similary, we arrive

$g\left(\widetilde{\mathrm{T}}_{E} G, F\right)=g\left(T_{E} G, F\right)+\eta(v G) g(h \varphi(v E), F)-\eta(h G) g(V \varphi(v E), v F)$

Then subctracting (12) from (13) we get equation (10). Similar to (10), we can prove (11).

Theorem 3.3 Let $\pi$ is a Riemannian submersion with QSNMC. Including basic tensors $\tilde{T}$ and $\tilde{A}$

and

$$
\tilde{T}_{U} W=\tilde{T}_{W} U+\eta(v W) h \varphi(v U)-\eta(v U) h \varphi(v W)
$$

$$
\tilde{A}_{X} Y=\tilde{A}_{Y} X+\eta(h Y) v \varphi(h X)-\eta(h X) V \varphi(h Y)
$$

for all $U, W \in \Gamma(V)$ and $X, Y \in \Gamma(H)$.

Proof. For all $U, W \in \Gamma(V)$, by using (8), we get

$\widetilde{\mathrm{T}}_{U} W=T_{U} W+\eta(v W) h \varphi(v U)+\eta(h W) v \varphi(v U)$

and

$\widetilde{\mathrm{T}}_{W} U=T_{W} U+\eta(v U) h \varphi(v W)+\eta(h U) v \varphi(v W)$

Considering that $T_{U} W=T_{W} U$ and $h W=h U=0$ is subtracted from (14) and (15), the desired is obtained.

Secondly for all $X, Y \in \Gamma(H)$ by using (9), we have

$\widetilde{\mathrm{A}}_{X} Y=A_{X} Y+\eta(h Y) v \varphi(h X)+\eta(v Y) h \varphi(h X)$

And

$\widetilde{\mathrm{A}}_{Y} X=A_{Y} X+\eta(h X) v \varphi(h Y)+\eta(v X) h \varphi(h Y)$

Similarly, considering that $\eta(v Y)=0 ; \eta(v X)=0, A_{X} Y=-A_{Y} X$, using (16) and (17) is added, the desired is obtained.

Theorem 3.4 Let $\pi$ is a Riemannian submersion with QSNMC. Then we have

$\widetilde{\nabla}_{V} W=\widehat{\nabla}_{V} W+\widetilde{\mathrm{T}}_{V} W-\eta(W) \varphi(V)$,

$\widetilde{\nabla}_{V} X=h \widetilde{\nabla}_{V} X+T_{V} X+\eta(X) \varphi V$,

$\widetilde{\nabla}_{X} Y=A_{X} Y+h \widetilde{\nabla}_{X} Y$,

$\widetilde{\nabla}_{X} V=A_{X} V+v \widetilde{\nabla}_{X} V+\eta(V) h \varphi(X)$

for all $U, W \in \Gamma(V)$ and $X, Y \in \Gamma(H)$.

Proof. Firstly, for all $U, W \in \Gamma(V)$, we have 
$\widetilde{\nabla}_{V} W=\nabla_{V} W+\eta(V) \varphi W$

or

$\nabla_{V} W=\widetilde{\nabla}_{V} W+\eta(V) \varphi W$

We know that,

$\nabla_{V} W=v \nabla_{V} W+h \nabla_{V} W$

Then using (3), we arrive

$\nabla_{V} W=v \widetilde{\nabla}_{V} W+\eta(V) v \varphi W+h \widetilde{\nabla}_{V} W+\eta(V) h \varphi W$

Hence proof can be complated placing (23) in (22) considering

$v \widetilde{\nabla}_{V} W=\widehat{\nabla}_{V} W$ and $h \widetilde{\nabla}_{V} W=T_{V} W$.

Similary, for all $V \in \Gamma(V)$ and $X \in \Gamma(H)$ by using (3), we have,

$\nabla_{V} X=v \widetilde{\nabla}_{V} X-\eta(X) v \varphi V+h \widetilde{\nabla}_{V} X-\eta(X) h \varphi V$.

Hence, we consider $v \widetilde{\nabla}_{V} X=\widetilde{T}_{V} X$ and $h \widetilde{\nabla}_{V} X=\tilde{A}_{V} X$. Then, (19) is achived.

On the other hand, for all $X, Y \in \Gamma(H)$ from (3) we arrive

$\nabla_{V} X=v \widetilde{\nabla}_{X} Y-\eta(Y) v \varphi X+h \widetilde{\nabla}_{X} Y-\eta(Y) h \varphi X$

If the expression (24), including $v \tilde{\nabla}_{X} Y=A_{X} Y$, which gives (20).

Finally $V \in \Gamma(V)$ and $X \in \Gamma(H)$ by using (3), we conclude,

$\nabla_{X} V=v \widetilde{\nabla}_{X} V-\eta(V) v \varphi X+h \widetilde{\nabla}_{X} Y-\eta(V) h \varphi X$

Then, including which gives (21).

\section{Curvature relations with respect to quarter-symmetric non-metric connection}

Finally, curvature properties of vertical and horizontal spaces of a Riemannian submersion defined by a quarter symmetric non-metric connection between two Riemannian manifolds are investigated.

Theorem 4.1 Let $\pi$ is a Riemannian submersion with QSNMC. We denote Riemanian curvatures of $M$ and any fibre $\left.\pi^{-1}(x), \hat{g}_{x}\right)$ by $\tilde{R}, \hat{R}$ respectively. Then we have

$$
\begin{aligned}
g(\tilde{R}(U, V) W, F) & =g(\hat{R}(U, V) W, F)-\eta\left(\widehat{\nabla}_{V} W\right) g(\varphi(U), F)+\eta\left(\widehat{\nabla}_{U} W\right) g(\varphi(V), F) \\
& +\eta\left(\widetilde{T}_{V} W\right) g(v \varphi(U), F)-\eta\left(\widetilde{T}_{U} W\right) g(v \varphi(V), F)-g\left(\widetilde{\nabla}_{U} \eta(W) \varphi(V), F\right)
\end{aligned}
$$




$$
+g\left(\widetilde{\nabla}_{V} \eta(W) \varphi(U), F\right)-\eta(W) g(\varphi([U, V]), F)
$$

And

$$
\begin{aligned}
& g(\tilde{R}(U, V) W, F)=g\left(\widetilde{T}_{U} \widehat{\nabla}_{V} W, X\right)-g\left(\widetilde{T}_{V} \widehat{\nabla}_{U} W, X\right)-\eta\left(\widehat{\nabla}_{V} W\right) g(\varphi(U), X) \\
& +\left(\widehat{\nabla}_{U} W\right) g(\varphi(V), X)+g\left(h \widetilde{\nabla}_{U} T_{V} W, X\right)-g\left(h \widetilde{\nabla}_{V} T_{U} W, X\right)+g\left(T_{U} \widetilde{T}_{V} W, X\right) \\
& \\
& -g\left(T_{V} \widetilde{T}_{U} W, X\right)-g\left(\widetilde{\nabla}_{U} \eta(W) \varphi(V), X\right)+g\left(\widetilde{\nabla}_{V} \eta(W) \varphi(U), X\right) \\
& \quad-g\left(\widetilde{T}_{[U, V]} W, X\right)+\eta(W) g(\varphi([U, V]), X)
\end{aligned}
$$

for all $U, V, W, F \in \Gamma(V)$ and $X \in \Gamma(H)$.

Proof. With respect to the quarter symmetric non-metric connection $M$ is the Riemann curvature tensor $\tilde{R}$ by

$\tilde{R}(U, V) W=\widetilde{\nabla}_{U} \widetilde{\nabla}_{V} W-\widetilde{\nabla}_{V} \widetilde{\nabla}_{U} W-\widetilde{\nabla}_{[U, V]} W$

Using (18) and (19), we can reach the following equation by direct operations

$$
\begin{aligned}
& \tilde{R}(U, V) W=\hat{R}(U, V) W+\widetilde{T}_{U} \widehat{\nabla}_{V} W-\widetilde{T}_{V} \widehat{\nabla}_{U} W-\eta\left(\widehat{\nabla}_{V} W\right) \varphi(U)+\eta\left(\widehat{\nabla}_{U} W\right) \varphi(V) \\
& \quad+h \widetilde{\nabla}_{U} T_{V} W-h \widetilde{\nabla}_{V} T_{U} W+T_{U} \widetilde{T}_{V} W-T_{V} \widetilde{T}_{U} W+\eta\left(\widetilde{T}_{V} W\right) v \varphi(U)-\eta\left(\widetilde{T}_{U} W\right) v \varphi(V) \\
& \quad-\widetilde{\nabla}_{U} \eta(W) \varphi(V)+\widetilde{\nabla}_{V} \eta(W) \varphi(U)-\widetilde{T}_{[U, V]} W+\eta(W) \varphi([U, V])
\end{aligned}
$$

If equation (28) is inner product with $F \in \Gamma(V)$, the equation (25) is obtained.

Similarly, if (28) equation is made with $X \in \Gamma(H)$ inner product (26) is obtained.

Theorem 4.2 Let $\pi$ is a Riemannian submersion with QSNMC. We denote Riemanian curvatures $M$ and $B$ by $\tilde{R}, R^{\prime}$ respectively. Then we have

$$
\begin{aligned}
g(\tilde{R}(X, Y) Z, K)= & g\left(R^{\prime}(X, Y) Z, K\right)-g\left(A_{X} K, A_{Y} Z\right)+g\left(A_{Y} K, A_{X} Z\right) \\
& +\eta\left(A_{Y} Z\right) g(h \varphi(X), K)-\eta\left(A_{X} Z\right) g(h \varphi(Y), K)
\end{aligned}
$$

and

$$
\begin{aligned}
g(\tilde{R}(X, Y) Z, U) & =g\left(v \widetilde{\nabla}_{X} A_{Y} Z, U\right)-g\left(v \widetilde{\nabla}_{Y} A_{X} Z, U\right)+g\left(A_{X} h \widetilde{\nabla}_{Y} Z, U\right) \\
& -g\left(A_{Y} h \widetilde{\nabla}_{X} Z, U\right)-g\left(T_{U} Z, A_{X} Y\right)-\eta(Z) g(v \varphi([X, Y]), U)
\end{aligned}
$$

for all $X, Y, Z, K \in \Gamma(H)$ and $U \in \Gamma(V)$.

Proof. For all $X, Y, Z, K \in \Gamma(H)$, using (27), (20) and (21) we have

$$
\begin{aligned}
\tilde{R}(X, Y) Z= & R^{\prime}(X, Y) Z+A_{X} A_{Y} Z-A_{Y} A_{X} Z+v \widetilde{\nabla}_{X} A_{Y} Z-v \widetilde{\nabla}_{Y} A_{X} Z+\eta\left(A_{Y} Z\right) h \varphi(X) \\
& -\eta\left(A_{X} Z\right) h \varphi(Y)+A_{X} h \widetilde{\nabla}_{Y} Z-A_{Y} h \widetilde{\nabla}_{X} Z-T_{[X, Y]} Z-\eta(Z) v \varphi([X, Y]) .
\end{aligned}
$$

Taking the inner product in (31) with K we obtain (29). In a similar way taking the inner product in (31) with U we obtain

$$
g(\tilde{R}(X, Y) Z, V)=g\left(v \widetilde{\nabla}_{X} A_{Y} Z, V\right)-g\left(v \widetilde{\nabla}_{Y} A_{X} Z, V\right)+g\left(A_{X} h \widetilde{\nabla}_{Y} Z, V\right)
$$




$$
-g\left(A_{Y} h \widetilde{\nabla}_{X} Z, V\right)-g\left(T_{[X, Y]} Z, V\right)-\eta(Z) g(v \varphi([X, Y]), V)
$$

Since $[X, Y]$ is vertical, we have $\widetilde{\nabla}_{X} Y=\widetilde{\nabla}_{Y} X$. On the other hand $T_{[X, Y]} Z=-2 T_{A_{X} Y} Z$ and from the property of metric $g\left(T_{[X, Y]} Z, U\right)=-g\left(2 T_{A_{X} Y} Z, U\right)=2 g\left(T_{U} Z, A_{X} Y\right)$ are obtained. So we arrive at the equation (30).

\section{References}

[1] Friedmann, A., Schouten, J.A., "Über die Geometrie der halbsymmetrischen Ü bertragung”, Math. Zeitschr. 21 (1924) : 211-223.

[2] Hayden, H.A., "Subspaces of a space with torsion", Proc. London Math. Soc. 34(1) (1932) : 27-50.

[3] Yano,K., “On semi symmetric metric connection”, Rev. Roumaine Math. Pures Appl.15 (1970), 1579-1586.

[4] Ayar, G., Demirhan, D., "Ricci solition on nearly Kenmotsu manifolds with semisymmetric metric connection”, Journal of Engineering Technology and Applied Sciences 4(3) (2019) : 131- 140.

[5] Altın, M., "Projective Curvature N(k) Contact metric manifold admitting semi-symetric non-metric connection”, Fundamental Journal of Mathematics and Applications 3(2) (2020) : 94-100.

[6] Chaubey, S. K., Ojha, R.H., "On semi-symmetric non-metric and quarter symmetric metric connections", Tensor N. S. 70(2) (2008) : 202-213.

[7] Golab, S., "On semi- symmetric metric and quarter symmetric linear connections", Tensor 29, (1975), 249-254.

[8] Dubey, A. K. Ojha, R. H. and Chaubey, S. K., "Some properties of quarter symmetric non-metric connection in a Kahler manifold”, Int. J. Contemp. Math.Sciences, 5(20), (2010), 1001-1007.

[9] Mishra, R. S. and Pandey, S. N., “On quarter symmetric metric F-connections”, Tensor N. S., 34, (1980), 1-7.

[10] Rastogi, S. C., "On quarter-symmetric metric connection”, C. R. Acad. Bulg. Sci. 31(8) (1978) : 811-814.

[11] Sarı,R. Akyol,M.A. Aksoy,E., "Some curvature properties of an S-manifold with a quarter symmetric non metric connection”, International Journal of Applied Mathematics and Statistic 56(3) (2017) : 93-102.

[12] O’Neill, B., “The fundamental equations of a submersion”, Michigan Math. J., 13, (1966), 459-469.

[13] Gray, A., "Pseudo Riemannian almost product manifold and submersions”, J. Math Metch. 16 (1967) : 715-737.

[14] Akyol M.A., Beyendi S., "Riemannian submersions endowed with a semi-symmetric nonmetric connection”, Konuralp Journal of Mathematics 6(1) (2018) : 188-193. 